\title{
Sr isotopes in the Orgueil CI meteorite: Chronology of early solar system hydrothermal activity
}

\author{
J D MACDOUGALL \\ Scripps Institution of Oceanography, La Jolla, CA 92093-0220, USA. \\ email: jdmacdougall@ucsd.edu
}

\begin{abstract}
New Sr isotopic analyses and calculated formation ages of carbonates from the Orgueil CI meteorite are reported. Among the samples analyzed in this work, dolomites give the youngest formation ages and may have been deposited intermittently starting near the time of parent body formation and continuing for at least $30 \mathrm{Ma}$. The Sr isotope data also suggest that breunnerites (Fe-Mn-Mg carbonates) crystallized after dolomite formation. Leaching experiments on bulk meteorite samples provide evidence for a very mobile, water soluble $\mathrm{Sr}$ reservoir in Orgueil that is characterized by extremely radiogenic $\mathrm{Sr}{ }^{87} \mathrm{Sr} /{ }^{86} \mathrm{Sr} \approx 0.81-$ 0.82). This unsupported Sr reflects recent element redistribution, possibly at the time of parent body breakup recorded by the $\sim 10$ Ma exposure age of Orgueil. The carbonate data in particular corroborate earlier indications that hydrothermal processes were among the earliest events to affect the CI parent body.
\end{abstract}

\section{Introduction}

The CI meteorites appear to be extensively altered mineralogically compared to their precursor material. However, because their relative bulk elemental abundances closely match those of the sun, they are also (somewhat paradoxically) believed to be little changed chemically. The original mineralogy of the CIs is not known with certainty, but it is generally accepted that the inferred mineralogical transformations occurred through the action of water, probably liquid water, within the CI parent body (e.g. DuFresne and Anders 1962; Richardson 1978; Kerridge and Bunch 1979). The mineral assemblage now observed in these meteorites includes a variety of phyllosilicates, sulfides and sulphates, magnetite, and carbonates-an assemblage that on the earth is typical of low temperature hydrothermal alteration of mafic material. Although these meteorites are brecciated to clast sizes that are frequently $<1 \mathrm{~mm}$, there is petrographic as well as mineralogical evidence for aqueous alteration, in the form of remnants of mineralized fractures filled with sulphates, or, more rarely, carbonates (Richardson 1978). Based on petrographic criteria, Richardson (1978) concluded that the carbonate vein fragments comprise the oldest generation of aqueous deposits now observable in the CI meteorites.

In addition to the phases noted above, the CI meteorites also contain small amounts of olivine and pyroxene that show no sign of interaction with hydrous fluids (Kerridge 1968; Reid et al 1970; Kerridge and Macdougall 1976). It is likely that these grains were added to the meteorite parent body after the main phase(s) of aqueous activity but before final lithification. A study by Goswami et al (1976) showed that in Orgueil, $\sim 15 \%$ of a group of 40 olivines examined for particle tracks exhibit evidence for irradiation with little or no shielding; considering both track and noble gas data, these authors concluded that the irradiated grains probably had a completely different pre-lithification history than the bulk meteorite. Whether the irradiation of these grains occurred in the regolith of the CI parent body, or in space before they were added to the parent body, is unknown. In any event, their addition must represent one of the last processes that occurred before consolidation of the CI parent body.

Carbonate minerals in Orgueil are the focus of the present paper. Although relatively rare, they have

Keywords. Orgueil; carbonate; Sr isotopes; hydrothermal; chronology. 
been the object of much attention because their chemical compositions provide clues to their formation environment, and they occur at grain sizes large enough to be amenable to hand picking and electron and ion microprobe analysis (Fredriksson and Kerridge 1988; Johnson and Prinz 1993; Riciputi et al 1994; Endress and Bischoff 1996). Dolomite dominates among the CI carbonates; in the most comprehensive study to date (Endress and Bischoff 1996), 85\% of all carbonates analyzed in 18 thin sections of four CI meteorites were dolomites. Breunnerite (ferroan magnesite) was found by these authors to be the next most abundant, followed by calcite at only about $1 \%$. These results are broadly similar to those of other workers, although the fraction of calcite observed by Endress and Bischoff (1996) is lower than found in some other studies. Numbers of grains, however, may not provide an especially accurate accounting of the importance of these phases. In Orgueil, dolomite and calcite occur as much smaller grains than breunnerite, typically $<100 \mu \mathrm{m}$ compared with breunnerite grains up to $1 \mathrm{~mm}$ and larger (Fredriksson and Kerridge 1988). Breunnerites also frequently exhibit well-formed crystal faces and they usually occur as isolated grains, whereas dolomite is typically anhedral and sometimes occurs as elongated clusters or fragments that have been interpreted as vein fragments (Richardson 1978). However, although Richardson (1978) also reported the presence of entire veins or veinlets in the sections he examined, later workers have not corroborated this observation, reporting instead only isolated carbonate grains and multi-grain clusters or fragments.

Ion probe data for carbonates in the $\mathrm{CM}$ carbonaceous chondrites suggest that calcite and dolomite in these meteorites formed from different fluids (Riciputi et al 1994), and, by inference, the same may be true for the CI meteorites. Some carbonate fragments in Orgueil contain at least two separate carbonate generations, which may be physically separated by a band of another phase, such as magnetite. This is a further indication that different fluids, possibly representing different hydrothermal episodes, were responsible for the formation of the different carbonates. Multiple stages of alteration are also suggested by the matrix mineralogy of Orgueil (Tomeoka and Buseck 1988).

The temperatures of carbonate deposition are not well known, although all estimates, both isotopic and those based on chemical composition, suggest that they were relatively low, probably well below $250^{\circ} \mathrm{C}$ (Clayton and Mayeda 1984; Johnson and Prinz 1993; Endress and Bischoff 1996). The heat energy necessary to drive hydrothermal processes in the CI parent body is believed to have come mainly from decay of short-lived radionuclides such as ${ }^{26} \mathrm{Al}$ (DuFresne and Anders 1962; Grimm and McSween 1989), and secondarily from impact processes (Grimm and McSween 1989). Oxygen isotope data also suggest a high water/rock ratio $(\sim 4: 1)$, at least at some stage of the aqueous alteration of Orgueil (Clayton and Mayeda 1984). This in turn implies that significant amounts of water flowed through the Orgueil parent material during alteration, presumably accompanied by a large mass flux of readily soluble species. Such a scenario is consistent with the generally accepted pervasive nature of CI meteorite alteration, but at odds with the observation that there is considerable chemical and mineralogical heterogeneity at the millimeter or smaller scale that suggests "local" variation in hydrothermal activity (McSween and Richardson 1977; Tomeoka and Buseck 1988; Endress and Bischoff 1996). This small-scale variability may have been produced during the waning stages of hydrothermal activity, after the main, pervasive, episode of fluid flow. One important caveat should be emphasized in this discussion: although Orgueil is by far the largest CI recovered (total mass $\sim 10 \mathrm{~kg}$ ), most of the petrographic, mineralogic, and chemical observations cited here were made on very small amounts of material, typically thin sections of a few $\mathrm{cm}^{2}$ or bulk samples weighing no more than a few grams, and often much less. Discussions of processes at the parent body scale therefore require very large extrapolation.

Proper understanding of the evolution of CI meteorites requires knowledge of the chronology of processes inferred to have affected these bodies. Petrographic observations provide a relative timescale for some events; for example, the observation that most carbonate veins have been completely disaggregated implies that brecciation either occurred entirely after their formation, or was partly coeval but continued after carbonate vein deposition. Chemical considerations and analogy with terrestrial processes suggest that there was a calcite-dolomite-sulphate depositional sequence. However, only radioactive decay processes offer the possibility of deducing the actual timescale for these events. Two approaches have been employed to date, both focused on carbonates: Mn-Cr (Endress et al 1996; Hutcheon and Phinney 1996) and Rb-Sr (Macdougall et al 1984) dating. Both of these methods require an accurate knowledge of the initial isotope ratio (radiogenic daughter/stable) at the time of formation of the CI parent body. Results from both types of study imply that the carbonates are ancient, formed within tens to at most a hundred million years or so after the parent body. In this paper, new $\mathrm{Sr}$ isotope data are presented for Orgueil. These data help to clarify some of the conclusions reached earlier about the carbonate formation processes.

\section{Methods}

As in our previous work (Macdougall et al 1984), the samples of Orgueil used in the experiments described here were generously provided by the late P. Pellas. 
Table 1. Sample details.

\begin{tabular}{|c|c|c|c|}
\hline Sample & Type & Weight & Treatment \\
\hline ORG-A-L1 & Water leach of bulk Orgueil fragment & $101.3 \mathrm{mg}$ & $\begin{array}{l}\text { Bulk sample leached in distilled water, } \\
\sim 44 \mathrm{hrs}\end{array}$ \\
\hline ORG-A-L2 & $0.5 \mathrm{~N} \mathrm{HCl}$ leach of solid residue from ORG-A-L1 & - & $\begin{array}{l}\text { Solid residue from ORG-A-L1 leached in } \\
0.5 \mathrm{~N} \mathrm{HCl}, \sim 40 \mathrm{~min}\end{array}$ \\
\hline ORG-A-L3 & $2 \mathrm{~N} \mathrm{HCl}$ leach of solid residue from ORG-A-L2 & - & $\begin{array}{l}\text { Solid residue from ORG-A-L2 leached in } \\
2 \mathrm{~N} \mathrm{HCl}, \sim 6 \mathrm{hr}\end{array}$ \\
\hline ORG-B-L1 & Water leach of bulk Orgueil fragment & $102.5 \mathrm{mg}$ & $\begin{array}{l}\text { Bulk sample leached in distilled water, } \\
\sim 44 \mathrm{hrs}\end{array}$ \\
\hline ORG-B-L2 & $0.5 \mathrm{~N} \mathrm{HCl}$ leach of solid residue from ORG-B-L1 & - & $\begin{array}{l}\text { Solid residue from ORG-B-L1 leached in } \\
0.5 \mathrm{~N} \mathrm{HCl}, \sim 60 \mathrm{~min}\end{array}$ \\
\hline ORG-B-L3 & $2 \mathrm{~N} \mathrm{HCl}$ leach of solid residue from ORG-A-L2 & - & $\begin{array}{l}\text { Solid residue from ORG-B-L2 leached in } \\
2 \mathrm{~N} \mathrm{HCl}, \sim 6 \mathrm{hr}\end{array}$ \\
\hline ORG-CA-1 & Single dolomite fragment from clast & $94 \mu \mathrm{g}$ & $\begin{array}{l}\text { Multiple distilled water washes, then } \\
\text { dissolved in } 1 \mathrm{~N} \mathrm{HCl}\end{array}$ \\
\hline ORG-CA-2 & Single dolomite fragment from clast & $48 \mu \mathrm{g}$ & $\begin{array}{l}\text { Multiple distilled water washes, then } \\
\text { dissolved in } 4.5 \mathrm{~N} \mathrm{HCl}\end{array}$ \\
\hline ORG-CA-3 & Breunnerite grains picked from clast & $795 \mu \mathrm{g}$ & $\begin{array}{l}\text { Multiple distilled water washes, then } \\
\text { dissolved in warm } 4.5 \mathrm{~N} \mathrm{HCl}\end{array}$ \\
\hline ORG-CA-4 & Dolomite grains from same clast as ORG-CA-2 & $56 \mu \mathrm{g}$ & $\begin{array}{l}\text { Multiple distilled water washes, then } \\
\text { dissolved in } 4.5 \mathrm{~N} \mathrm{HCl}\end{array}$ \\
\hline ORG-CA-5 & Breunnerite grains from same clast as ORG-CA-3 & $2.51 \mathrm{mg}$ & $\begin{array}{l}\text { Multiple distilled water washes, } 0.5 \mathrm{~N} \mathrm{HCl} \\
\text { wash, then dissolved in warm } 4.5 \mathrm{~N} \mathrm{HCl}\end{array}$ \\
\hline
\end{tabular}

All were coherent fragments with no visible traces of the contaminating terrestrial material sometimes found mixed with Orgueil samples. Two types of analysis were carried out. First, a series of leaching experiments was conducted on small Orgueil fragments without fusion crusts (see table 1). Two fragments, each weighing approximately $100 \mathrm{mg}$, were separately and sequentially leached first in distilled water, then $0.5 \mathrm{~N} \mathrm{HCl}$, and finally $2 \mathrm{~N} \mathrm{HCl}$. The leach solutions from each step were removed, spiked and analyzed for $\mathrm{Rb}$ and $\mathrm{Sr}$ concentrations and $\mathrm{Sr}$ isotopic composition. Slight effervescence occurred when $\mathrm{H}_{2} \mathrm{O}$ was initially added to these fragments, and the fragments disintegrated into smaller pieces. The water leach solution remained very clear and uncoloured, while the $0.5 \mathrm{~N} \mathrm{HCl}$ leach was slightly cloudy and brownish-yellow, and the $2 \mathrm{~N} \mathrm{HCl}$ leach clear and bright yellow. After the supernatant leach solution was removed at each step, the residual solid was rinsed several times in distilled water, and the rinse added to the leach solution.

Other fragments of Orgueil were examined microscopically for carbonate grains and clasts. Three multicrystal clasts, two identified as being predominantly dolomite and one breunnerite, were chosen for analysis. Mineral identification was based on energy dispersive X-ray spectra obtained from a few small grains from each clast, examined with the scanning electron microscope; it was assumed that these grains were representative of the whole clast. Crystals from these clasts that appeared to be inclusion-free and without significant amounts of adhering or intergrown matrix were picked for analyses. All were sonified and rinsed repeatedly in distilled water prior to dissolution for Sr isotope analysis. In addition, breunnerite sample
CA-5 (see table 1) was also sonified for 5 minutes in $0.5 \mathrm{~N} \mathrm{HCl}$ prior to dissolution.

$\mathrm{Sr}$ isotopic data are reported relative to a value of $0.710260 \pm 0.000024$ for NBS 987, where the uncertainty is the total range of standard values over the course of these analyses. Procedural blanks averaged $3 \mathrm{pg}$ for $\mathrm{Rb}$ and $15 \mathrm{pg}$ for $\mathrm{Sr}$ during this work; all reported concentration and isotope data are blankcorrected where necessary.

\section{Results}

\subsection{Bulk Orgueil leaching experiments}

Data from the leaching experiments (tables 1 and 2) turned out to be especially interesting. In earlier work, Fredriksson and Kerridge (1988) showed that most of the $\mathrm{Na}, \mathrm{K}$ and $\mathrm{SO}_{3}^{=}$, as well as about $\frac{1}{2}$ of the $\mathrm{Ca}$ and more than $10 \%$ of the $\mathrm{Mg}$ in Orgueil are water soluble. Their data were based on extraction of a $1 \mathrm{gm}$ bulk sample with boiling water. The data reported here, based on room-temperature extraction using distilled water, indicate that large fractions of the $\mathrm{Sr}$ and $\mathrm{Rb}$ are also water-soluble, as might be expected from the major element data. Although a bulk rock analysis was not carried out in this work, data in the literature can be used to estimate the fraction of $\mathrm{Sr}$ and $\mathrm{Rb}$ removed by the leaching treatments. Anders and Ebihara (1982) indicate that the CI meteorites contain about $7.91 \mathrm{ppm}$ Sr and $2.30 \mathrm{ppm} \mathrm{Rb;} \mathrm{similar} \mathrm{results} \mathrm{were}$ obtained by Mittlefehldt and Wetherill (1979) for three separate samples of Orgueil. The elemental data for the leach solutions are given in table 2, as concentrations calculated using the original sample weight. It should be noted that these are approximate 
Table 2. Isotope and concentration data.

\begin{tabular}{lcccl}
\hline Sample & ${ }^{87} \mathrm{Sr} /{ }^{86} \mathrm{Sr}_{m}$ & ${ }^{87} \mathrm{Sr} /{ }^{86} \mathrm{Sr}_{c}$ & $\begin{array}{c}\mathrm{Sr} \\
(\mathrm{ppm})\end{array}$ & $\begin{array}{c}\mathrm{Rb} \\
(\mathrm{ppm})\end{array}$ \\
\hline ORG-A-L1 & $0.818913 \pm 24$ & $0.818913 \pm 24$ & 2.70 & 1.1 \\
ORG-A-L2 & $0.735634 \pm 24$ & $0.735634 \pm 24$ & 3.90 & 0.71 \\
ORG-A-L3 & $0.743264 \pm 30$ & $0.743264 \pm 30$ & 0.30 & 0.3 \\
ORG-B-L1 & $0.812281 \pm 24$ & $0.812281 \pm 24$ & 2.70 & 1.03 \\
ORG-B-L2 & $0.732276 \pm 24$ & $0.732276 \pm 24$ & 4.07 & 0.74 \\
ORG-B-L3 & $0.749362 \pm 24$ & $0.749362 \pm 24$ & 0.20 & 0.34 \\
ORG-CA-1 & $0.699366 \pm 58$ & $0.699315 \pm 62$ & 39 & 0.002 \\
ORG-CA-2 & $0.699075 \pm 56$ & $0.699034 \pm 60$ & 98 & 0.10 \\
ORG-CA-3 & $0.70121 \pm 13$ & $0.70101 \pm 17$ & 0.99 & 0.019 \\
ORG-CA-4 & $0.699157 \pm 56$ & $0.69911 \pm 6$ & 73 & 0.39 \\
ORG-CA-5 & $0.70084 \pm 12$ & $0.70075 \pm 14$ & 0.80 & 0.005 \\
\hline Con
\end{tabular}

Concentrations are corrected for procedural blanks of $15 \mathrm{pg}$ (Sr) and $3 \mathrm{pg}(\mathrm{Rb})$. Subscripts " $m$ " and "c" for Sr isotope ratios refer to measured and blank-corrected, respectively. ${ }^{87} \mathrm{Sr} /{ }^{86} \mathrm{Sr}=$ 0.712 was used for the blank composition. Uncertainties in the isotope ratios refer to the final digits and are the larger of the total range for standards during this work $( \pm 0.000024)$, or the single-run uncertainty.

concentrations, because samples were not dried before weighing; the CI meteorites are known to change weight with variations in ambient humidity. Note also that the mobile nature of $\mathrm{Rb}$ and/or $\mathrm{Sr}$ is implied by the "young" model ages obtained by Mettlefehlt and Wetherill (1979) in their study of Orgueil. These authors found that ${ }^{87} \mathrm{Sr} /{ }^{86} \mathrm{Sr}$ in the small fragments they analyzed was always "too low" for the measured $\mathrm{Rb} / \mathrm{Sr}$, assuming an age for Orgueil near $4.55 \mathrm{Ga}$. It is clear that at some scale - and probably at a variety of scales - Orgueil has been an open system.

The leaching data show that the combined water and $\mathrm{HCl}$ leaches together extract about $90 \%$ of the $\mathrm{Sr}$ and $\mathrm{Rb}$ contained in the meteorite. The remaining $10 \%$ must be retained in the undissolved residue, composed primarily of phyllosilicates and magnetite. The amounts of $\mathrm{Sr}$ and $\mathrm{Rb}$ extracted in comparable leach steps are very similar for the two Orgueil samples (fragments ORG-A and ORG-B), except for $\mathrm{Sr}$ in the final leach of ORG-B, which is significantly lower than for the same step in ORG-A. Especially interesting are the data for the water leaches. Roughly a third of the $\mathrm{Sr}$ in Orgueil, and almost $50 \%$ of the Rb, are extracted in a simple water leach at room temperature. The only readily identifiable phase in this meteorite that is water soluble is $\mathrm{Mg}$ sulphate, which may contain variable amounts of $\mathrm{Na}, \mathrm{Ni}, \mathrm{Ca}, \mathrm{Fe}$ and $\mathrm{Mn}$ (Fredriksson and Kerridge 1988). It is likely that there are also very fine-grained soluble species on grain surfaces distributed throughout the meteorite, as suggested by earlier work (Macdougall et al 1984) in which it was observed that water rinses of hand-picked carbonate grains contained large amounts of $\mathrm{Sr}$ with isotopic ratios very different from the grains themselves.

The $\mathrm{Sr}$ isotopic compositions of the two water leaches are similar and very radiogenic (table 2 ). They are significantly higher than that of the bulk meteorite, which has ${ }^{87} \mathrm{Sr} /{ }^{86} \mathrm{Sr} 0.753$ (Mittlefehldt and Wetherill 1979). The high values in the leach solutions are consistent with our earlier data on $\mathrm{Sr}$ from the water rinses of picked carbonate grains; these had values of 0.85337 and 0.80592 for ${ }^{87} \mathrm{Sr} /{ }^{86} \mathrm{Sr}$ (Macdougall et al 1984). In spite of the fact that a large amount of $\mathrm{Rb}$ was also extracted in the bulk sample water leaches, the radiogenic $\mathrm{Sr}$ is unsupported by the $\mathrm{Rb}$ contained in the leaches. About 1.6 to 1.7 times the observed $\mathrm{Rb}$ amounts would be required to produce the measured $\mathrm{Sr}$ isotopic ratios over $4.56 \mathrm{Ga}$. This indicates either that there has been a relatively recent redistribution of $\mathrm{Rb}$ and $\mathrm{Sr}$ in the meteorite, so that some Rb now resides in a different phase than its daughter $\mathrm{Sr}$, or that some of the soluble $\mathrm{Rb}$ was transferred to the phyllosilicate matrix minerals during the leaching process. The fact that one of the two carbonate grain rinses measured in our earlier investigation (Macdougall et al 1984) - which had no contact with matrix phyllosilicates - had ${ }^{87} \mathrm{Rb} /{ }^{86} \mathrm{Sr}$ essentially equivalent to those of the two water leaches discussed in the present work suggests that the unsupported radiogenic $\mathrm{Sr}$ is not an artifact of sample processing, but is indeed unsupported in situ in Orgueil.

The first $\mathrm{HCl}$ leaches $(0.5 \mathrm{~N})$ of the residues from the water leaching experiment contained even more $\mathrm{Sr}$ than the water leach, about $50 \%$ of the total meteorite $\mathrm{Sr}$, but less Rb (table 2). These acidic leaches presumably dissolve $\mathrm{Ca}$ carbonate, Ca sulphate, and at least partly dissolve dolomite and breunnerite. Consistent with this scenario, ${ }^{87} \mathrm{Sr} /{ }^{86} \mathrm{Sr}$ in these solutions is lower than in the bulk meteorite, and also is the lowest of the three leach treatments. The final $(2 \mathrm{~N}) \mathrm{HCl}$ leach must continue to dissolve remaining carbonates, and also must extract some Sr (and $\mathrm{Rb}$ ) from phyllosilicates and other matrix minerals. Its ${ }^{87} \mathrm{Sr} /{ }^{86} \mathrm{Sr}$ is higher than the $0.5 \mathrm{~N} \mathrm{HCl}$ leach, but is lower than that of the bulk meteorite. The $\mathrm{HCl}$ leaches also reflect the redistribution of $\mathrm{Rb}$ and $\mathrm{Sr}$ evident in the water leach results.

\subsection{Dolomites and breunnerites}

Elemental and isotopic data for the dolomites and breunnerites are given in table 2. As already mentioned, all data are blank corrected, where necessary, for $3 \mathrm{pg} \mathrm{Rb}$ and $15 \mathrm{pg} \mathrm{Sr}$ blanks. While the $\mathrm{Sr}$ data in table 2 are probably robust, the $\mathrm{Rb}$ concentrations may not be accurate because in most cases the total amounts analyzed are no larger than a few times the blank levels. Thus precise knowledge of the actual blank amount in each sample would be required for accurate data. During this work, procedural blanks for $\mathrm{Rb}$ averaged near $3 \mathrm{pg}$, but occasionally significantly higher blanks occurred, probably due to scratched beakers, which are difficult to clean, or similar problems. There is also the possibility that the washing procedure did 
not always entirely remove matrix $\mathrm{Rb}$ from the surfaces of these low-Rb grains. Thus the $\mathrm{Rb}$ concentrations in table 2 should be treated as upper limits. This is emphasized by the fact that initial ${ }^{87} \mathrm{Sr} /{ }^{86} \mathrm{Sr}$ ratios (at $4.56 \mathrm{Ga}$ ), calculated using the measured ${ }^{87} \mathrm{Rb} /{ }^{86} \mathrm{Sr}$ values, are lower than the presumed initial solar system value of 0.69897 (Lugmair and Galer 1992) for the three carbonates with highest Rb contents (CA-2, CA-3 and CA-4).

$\mathrm{Sr}$ concentrations in the breunnerites are clearly much lower than in the dolomites (table 2), as already noted by Macdougall et al (1984). In the dolomite, Sr is quite variable and ranges from 39 to $98 \mathrm{ppm}$, while both breunnerite samples have $\mathrm{Sr}<1 \mathrm{ppm}$. Note that the $\mathrm{Sr}$ isotopic ratios are higher for breunnerite than for dolomite. It is conceivable that this is in part due to incomplete removal of the surface-correlated, easily leachable radiogenic $\mathrm{Sr}$ discussed above, although sample ORG-CA-5 was the largest of all carbonate samples measured, and therefore, in spite of its low $\mathrm{Sr}$ concentration, contained a significant total amount of Sr. Furthermore, this sample was rinsed with $0.5 \mathrm{~N}$ $\mathrm{HCl}$ in addition to water. Thus the effect of nonindigenous radiogenic $\mathrm{Sr}$ should be minimized in this sample, yet its ${ }^{87} \mathrm{Sr} /{ }^{86} \mathrm{Sr}$ is still higher than those measured in any of the dolomites.

Time scales for formation of the carbonates are given in table 3 and shown in figure 1 calculated assuming an initial ratio for Orgueil of 0.69897 and using the average ${ }^{87} \mathrm{Rb} /{ }^{86} \mathrm{Sr}$ for bulk Orgueil (0.87282) reported by Mittlefehldt and Wetherill (1979). They are also calculated using the measured Sr isotopic ratios rather than age-corrected values, because, as explained above, the measured $\mathrm{Rb}$ concentrations are upper limits and in some cases appear to be too high. Thus the ages themselves are upper limits. The data indicate that some carbonates were precipitated within a few million years of Orgueil's formation, and that there may be a significant gap (tens of millions of years) between dolomite and breunnerite formation. Note that for the two carbonates with lowest measured ${ }^{87} \mathrm{Rb} /{ }^{86} \mathrm{Sr}$, formation times are also given using age-

Table 3. Formation times for Orgueil carbonates.

\begin{tabular}{lccc}
\hline Sample & Mineral & ${ }^{87} \mathrm{Sr} /{ }^{86} \mathrm{Sr}_{c}$ & $\Delta t(\mathrm{Ma})$ \\
\hline CA-1 & Dolomite & $0.699315 \pm 62$ & $28 \pm 5$ \\
& & $0.699304 \pm 62$ & $27 \pm 5$ \\
CA-2 & Dolomite & $0.699034 \pm 60$ & $5.2 \pm 4.8$ \\
CA-4 & Dolomite & $0.699110 \pm 60$ & $11.3 \pm 4.8$ \\
CA-3 & Breunnerite & $0.70101 \pm 17$ & $164 \pm 14$ \\
CA-5 & Breunnerite & $0.70075 \pm 14$ & $143 \pm 12$ \\
& & $0.69965 \pm 14$ & $55 \pm 12$
\end{tabular}

As in table 2, the subscript "c" refers to blank-corrected $\mathrm{Sr}$ isotope data. For CA-1 and CA-5, the second line for each entry gives the age-corrected $(4.56 \mathrm{Ga})$ isotope ratio, calculated using the measured ${ }^{87} \mathrm{Rb} /{ }^{86} \mathrm{Sr}$. The formation time, $\Delta t$, is calculated for an initial ratio of 0.69897 (Lugmair and Galer 1992).

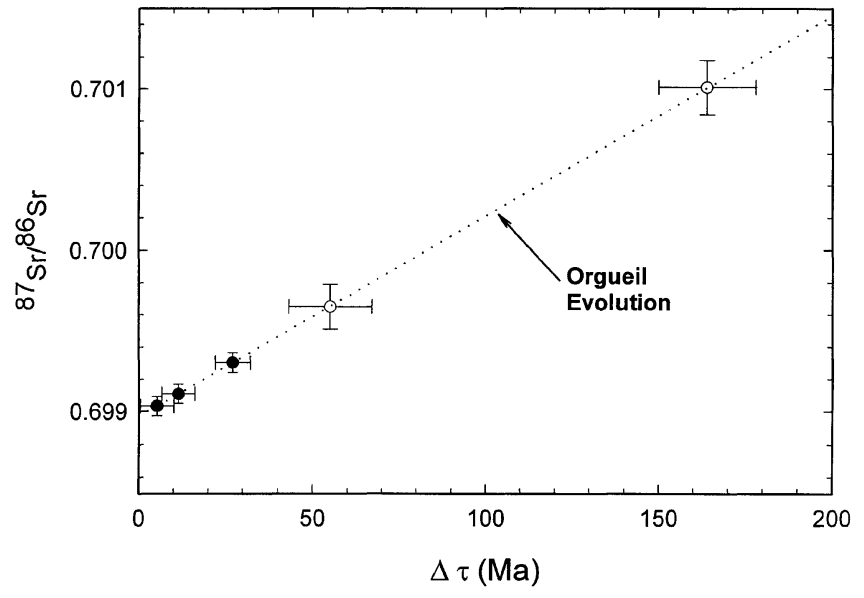

Figure 1. Isotope data from table 3 plotted along the Orgueil evolution line. Filled circles are dolomite data; open circles are breunnerite. See text for discussion of the formation ages $(\Delta \tau)$.

corrected $(4.56 \mathrm{Ga}) \mathrm{Sr}$ isotope ratios. Although these ages are slightly smaller than the uncorrected ones, they do not change the basic conclusion that there is a time difference between dolomite and breunnerite formation.

\section{Discussion}

The very high $\mathrm{Rb} / \mathrm{Sr}$ ratio in the CI meteorites provides high time resolution for events early in their history, provided that initial ratios for these events can be determined accurately. The carbonates, with very low $\mathrm{Rb} / \mathrm{Sr}$ and thus minimal ingrowth of radiogenic Sr since their formation, are ideal phases for such chronology. The concept is very simple for such phases: formation ages are simply the times required to acquire the measured (or initial) ${ }^{87} \mathrm{Sr} /{ }^{86} \mathrm{Sr}$ ratio in the carbonate given the bulk Orgueil ${ }^{87} \mathrm{Rb} /{ }^{86} \mathrm{Sr}$ and initial ${ }^{87} \mathrm{Sr} /{ }^{86} \mathrm{Sr}$. The possibility of determining the formation times for carbonates in the CI meteorites using $\mathrm{Sr}$ isotopes was first explored by Macdougall et al (1984); the main conclusion reached by these authors was that the carbonates in Orgueil formed very early in the meteorite's history. Formation times calculated for the samples they analyzed ranged from essentially contemporaneous with meteorite assembly to several hundred million years after formation. Very early formation of carbonates was corroborated by Endress et al (1996) and Hutcheon and Phinney (1996), who used ion probe techniques to measure $\mathrm{Cr}$ isotopes in dolomites and breunnerites from Orgueil. Both groups found ${ }^{53} \mathrm{Cr}$ excesses in Mn-rich carbonates, produced et al by short-lived ${ }^{53} \mathrm{Mn}$, which has a half life of only 3.7 Ma. The new results presented here also corroborate the earlier data, and they give additional information about both the soluble phases in Orgueil, and the relationship between the two major carbonate types, dolomite and breunnerite. 
As already discussed, the carbonate formation times given in table 3 and illustrated in figure 1 must be treated as upper limits because of the possibility that small amounts of non-carbonate radiogenic Sr were included in the analyses, and the fact that age corrections are only possible for two of the carbonates.

Dolomites CA-2 and CA-3 are both from the same carbonate clast, and their calculated formation times overlap within the experimental uncertainties. Since virtually all experimental factors affecting the calculated ages tend to increase them, it is reasonable to consider the smaller age as being the more accurate. Alternatively there could be more than one generation of carbonate within the same clast, as described by Endress and Bischoff (1996). Regardless, the data for CA-2 indicate that hydrothermal activity and carbonate precipitation began within a few million years of formation of the Orgueil parent body; in fact, at the lower end of the experimental uncertainty range, $\Delta t$ is only $0.4 \mathrm{Ma}$. The formation time for CA-2 is consistent with those calculated by Endress et al (1996), who measured five dolomite fragments, all with ${ }^{53} \mathrm{Cr}$ excesses, and concluded that they formed within $20 \mathrm{Ma}$ of the earliest solar system objects known (Allende calcium-aluminum rich inclusions). Sample CA-1 has a significantly larger calculated formation time, and the $\mathrm{Sr}$ isotope data presented here therefore suggest that dolomite formation could have extended over a time span of nearly $30 \mathrm{Ma}$. An important test of this conclusion will be further $\mathrm{Cr}$ isotope analyses of a range of petrographically documented dolomites. Grains formed $30 \mathrm{Ma}$ or longer after parent body formation should have very small or no ${ }^{53} \mathrm{Cr}$ excess, even for those with high $\mathrm{Mn} / \mathrm{Cr}$.

The breunnerite samples, both from the same clast, have much higher measured ${ }^{87} \mathrm{Sr} /{ }^{86} \mathrm{Sr}$ than the dolomites. Like the dolomites CA-2 and CA-4, their measured isotopic compositions and calculated formation times overlap (barely) within the experimental uncertainty (table 3 ). The rubidium content of sample CA-3 is clearly "too high", and results in an unacceptably low initial ratio if the isotope ratio for this sample is age-corrected. However, the data for CA-5 which was acid rinsed before dissolution - indicate a much lower indigenous Rb content. Even so, the agecorrected initial ratio for this sample is higher than those of the dolomites, and the calculated formation time $(55 \mathrm{Ma})$ is also. This does suggest that the breunnerites are significantly older than the dolomites. On the other hand, Hutcheon and Phinney (1996) measured $\mathrm{Cr}$ isotopes in several breunnerites with high $\mathrm{Mn} / \mathrm{Cr}$, and estimated that they formed within 12-16 Ma of Orgueil parent body formation. This is clearly earlier than the $\mathrm{Sr}$ isotope data for CA- 5 would suggest, but it is also considerably older than the youngest formation age determined for dolomite in this work. Again, further ion probe Cr isotope studies, with the potential for higher time resolution than is possible with $\mathrm{Sr}$ isotopes, may help to determine the variability of breunnerite ages more accurately.

The results of the bulk-meteorite leaching experiments are particularly intriguing, especially the data from the water leaches (table 2). They indicate that there is a large, very radiogenic Sr component in Orgueil that is readily soluble, and that has ${ }^{87} \mathrm{Sr} /{ }^{86} \mathrm{Sr}$ significantly greater than the bulk meteorite. What is the origin of this component? It is possible, but unlikely, that it is a contaminant. ${ }^{87} \mathrm{Sr} /{ }^{86} \mathrm{Sr}$ is so high in this component that most terrestrial reservoirs - with the exception of old, high $\mathrm{Rb} / \mathrm{Sr}$ crustal rocks - are ruled out as a source. The radiogenic Sr is apparently pervasively present in Orgueil, as is shown by the fact that in our earlier investigations (Macdougall et al 1984) the same component was encountered on the surfaces of picked carbonate grains. It therefore does not appear to be associated with a specific phase. It is well known that $\mathrm{Mg}$ sulphate crystals grow on freshly exposed surfaces of Orgueil, especially in humid environments, and presumably the soluble $\mathrm{Sr}$ is part of this mobile material. Its Sr isotopic composition, however, places some constraints on its history. It dictates that until recently, this $\mathrm{Sr}$ reservoir had $\mathrm{Rb} /$ Sr much higher than the bulk meteorite. It is likely that it represents the final, interstitial residue of the hydrothermal fluids that ultimately produced the mineral assemblage now present in Orgueil. The "orphan" nature of the radiogenic Sr, however, indicates that there has been recent redistribution of at least some of this residue. In particular, some of the $\mathrm{Rb}$ in the water soluble component became fixed in less-soluble phases. The timing of this redistribution cannot be determined accurately from the $\mathrm{Sr}$ isotope data, but it must be recent to explain the very high ${ }^{87} \mathrm{Sr} /{ }^{86} \mathrm{Sr}$. The cosmic ray exposure age of Orgueil is $\sim 10$ Ma (Jeffery and Anders 1970; Kerridge et al 1979), so it is conceivable that parent body breakup is the event reflected by these data.

\section{Conclusions}

The new $\mathrm{Sr}$ isotope data reported here for Orgueil carbonates and bulk meteorite leaches corroborate earlier indications that the carbonates were deposited by very early hydrothermal activity within the CI parent body, and that some very recent (probably within the past $10 \mathrm{Ma}$ or so) chemical processing has also affected element distribution within Orgueil. The earliest deposition time recorded in the samples analyzed in this work is for dolomite CA-2, which has very low ${ }^{87} \mathrm{Sr} /{ }^{86} \mathrm{Sr}$ indicating crystallization $\leq 5.2 \mathrm{Ma}$ after parent body formation, assuming an initial ratio of 0.69897 . The data also suggest that carbonate deposition continued over a time span of as much as several tens of millions of years, and that the $\mathrm{Fe}-\mathrm{Mn}-\mathrm{Mg}$ carbonates were mostly formed after 
dolomite deposition. Thus hydrothermal activity was an early and long-lasting feature of the CI parent body.

\section{Postscript}

This paper is dedicated to Professor K Gopalan on the occasion of his retirement from the National Geophysical Research Institute. Early in his career, K Gopalan, together with G Wetherill, wrote a series of papers on the Rb-Sr ages of various chondritic meteorite groups (Gopalan and Wetherill 1968, 1969, 1970, 1971). They were, in their time, groundbreaking contributions, and the papers are much cited in the literature. Some thirty years later our analytical capabilities are greatly improved, but the general approach is not radically different. The present manuscript adds to those earlier papers of Gopalan and Wetherill (and the papers of many others who have published on this topic in the interim) by presenting a Rb-Sr study of the CI meteorite Orgueil.

It has been my privilege to work with K Gopalan periodically over a substantial portion of his careernot, as it turns out, on meteorites, but rather on the isotopic geochemistry and geochronology of Indian rocks. This collaboration has been fruitful, and, for me at least, enlightening. It has been a scientific adventure, but it has been made even more enjoyable by countless adventures of other sorts on Indian highways, in Indian airports, cities and guesthouses, and in the Indian countryside.

The sciences, especially the Earth Sciences, are international endeavours, but it is only through the efforts of perceptive scientists such as K. Gopalan that the potential of international collaborations can be fully realized. So it is to be hoped that his retirement will be so in name only.

\section{Acknowledgments}

The late P Pellas generously supplied samples of Orgueil for this study. C MacIsaac and D Haffner helped with various aspects of the work. I am grateful to NASA and the U.S. National Science Foundation for long term support of the La Jolla Isotope Geology and Geochronology Laboratory.

\section{References}

Anders E and Ebihara M 1982 Solar-system abundances of the elements; Geochim. Cosmochim. Acta 46 2363-2380.

Clayton R N and Mayeda T K 1984 The oxygen isotope record in Murchison and other carbonaceous chondrites; Earth Planet. Sci. Lett. 67 151-161.

DuFresne E R and Anders E 1962 On the chemical evolution of the carbonaceous chondrites; Geochim. Cosmochim. Acta $\mathbf{2 6}$ 1085-1114.
Endress M and Bischoff A 1996 Carbonates in CI chondrites: Clues to parent body evolution; Geochim. Cosmochim. Acta 60, 489-507.

Endress M, Zinner E and Bischoff A 1996 Early aqueous activity on primitive meteorite parent bodies; Nature 379 701-703.

Fredriksson K and Kerridge J F 1988 Carbonates and sulfates in the CI chondrites: Formation by aqueous activity on the parent body; Meteoritics 23 35-44.

Gopalan K and Wetherill G W 1968 Rubidium-strontium age of hypersthene (L) chondrites; J. Geophys. Res. 73 7133-7136.

Gopalan K and Wetherill G W 1969 Rubidium-strontium age of amphoterite (LL) chondrites; J. Geophys. Res. 74 4349-4358.

Gopalan K and Wetherill G W 1970 Rubidium-strontium studies on enstatite chondrites: Whole meteorite and mineral isochrons; J. Geophys. Res. 75 3457-3467.

Gopalan K and Wetherill G W 1971 Rubidium-strontium studies on enstatite chondrites: Whole meteorite and mineral isochrons; J. Geophys. Res. 76 8484-8492.

Goswami J N, Hutcheon I D and Macdougall J D 1976 Microcraters and solar flare tracks in crystals from carbonaceous chondrites and lunar breccias; Proc. Lunar Sci. Conf. 7 543-562.

Grimm R E and McSween H Y 1989 Water and the thermal evolution of carbonaceous chondrite parent bodies; Icarus $\mathbf{8 2}$ 244-280.

Hutcheon, I D and Phinney D L 1996 Radiogenic ${ }^{53} \mathrm{Cr}^{*}$ in Orgueil carbonates: Chronology of aqueous activity on the CI parent body; Lunar. Planet. Sci. XXVII, 577-578. LPI, Houston.

Jeffery P M and Anders E 1970 Primordial noble gases in separated meteoritic minerals-I; Geochim. Cosmochim. Acta 34 1175-1198.

Johnson C A and Prinz M 1993 Carbonate compositions in C M and CI chondrites, and implications for aqueous alteration; Geochim. Cosmochim. Acta 57 2843-2852.

Kerridge J F 1968 Occurrence of olivine in a Type I carbonaceous meteorite; Nature 217 729-730.

Kerridge J F and Bunch T E 1979 Aqueous alteration on asteroids: Evidence from carbonaceous meteorites. In Asteroids (ed.) T. Gehrels, (Tucson, Univ. Arizona Press) 745-764.

Kerridge J F and Macdougall J D 1976 Mafic silicates in the Orgueil carbonaceous meteorite; Earth Planet. Sci. Lett. 29 341-348.

Kerridge J F, Macdougall J D and Marti K 1979 Clues to the origin of sulfide minerals in CI chondrites; Earth Planet. Sci. Lett. 43 359-367.

Lugmair G and Galer S J G 1992 Age and isotopic relationships among the angrites Lewis Cliff 86010 and Angra dos Reis; Geochimica Cosmochimica Acta 56, 1673-1694.

Macdougall J D, Lugmair G W and Kerridge J F 1984 Early solar system aqueous activity: $\mathrm{Sr}$ isotope evidence from the Orgueil CI meteorite; Nature 307 249-251.

McSween H Y and Richardson S M 1977 The composition of carbonaceous chondrite matrix; Geochim. Cosmochim. Acta 41 1145-1161.

Mittlefehldt D and Wetherill G W 1979 Rb-Sr studies of CI and CM chondrites; Geochim. Cosmochim. Acta 43 201-206.

Reid A M, Bass M N, Fujita H, Kerridge J F and Fredriksson K 1970 Olivine and pyroxene in the Orgueil meteorite; Geochim. Cosmochim. Acta 34 1253-1255.

Richardson S M 1978 Vein formation in the C1 carbonaceous chondrites; Meteoritics 13 141-159.

Riciputi L R, McSween H Y, Johnson C A and Prinz M 1994 Minor and trace element concentrations in carbonates of carbonaceous chondrites, and implications for the compositions of coexisting fluids; Geochim. Cosmochim. Acta $\mathbf{5 8}$ 1343-1351.

Tomeoka K and Buseck P R 1988 Matrix mineralogy of the Orgueil CI carbonaceous chondrite; Geochim. Cosmochim. Acta 52 1627-1640. 